\title{
How gender influence the health state? A cross sectional study in an endocrine setting
}

\author{
Marta Bianchini \\ Istituto Regina Elena
}

\section{Giulia Puliani}

Universita degli Studi di Roma La Sapienza Dipartimento di Medicina Sperimentale

Alfonsina Chiefari

Istituto Regina Elena

\section{Rosa Lauretta}

Istituto Regina Elena

Marilda Mormando

Istituto Regina Elena

Agnese Barnabei

Istituto Regina Elena

Irene Terrenato

Istituto Regina Elena

Marialuisa Appetecchia ( $\square$ marialuisa.appetecchia@ifo.gov.it)

Istituto Regina Elena IRCCS https://orcid.org/0000-0002-2124-1335

\section{Research article}

Keywords: gender medicine, lifestyle, endocrine disease, oncological comorbidities, determinants of health.

Posted Date: November 10th, 2020

DOI: https://doi.org/10.21203/rs.3.rs-103184/v1

License: (c) (i) This work is licensed under a Creative Commons Attribution 4.0 International License.

Read Full License 


\section{Title page}

2 How gender influence the health state? A cross sectional study in an endocrine setting

3 Marta Bianchini $^{1}$, Giulia Puliani ${ }^{1,2}$, Alfonsina Chiefari ${ }^{1}$, Rosa Lauretta ${ }^{1}$, Marilda Mormando ${ }^{1}$,

$4 \quad$ Agnese Barnabei ${ }^{1}$, Irene Terrenato ${ }^{3}$ and Marialuisa Appetecchia ${ }^{1 *}$

$5{ }^{1}$ Oncological Endocrinology Unit, IRCCS Regina Elena National Cancer Institute, Via Elio

6 Chianesi 53, 00144, Rome, Italy;

$7 \quad 2$ Department of Experimental Medicine, Sapienza University of Rome; Viale Regina Elena 324,

$8 \quad$ 00161, Rome, Italy;

$9{ }^{3}$ Biostatistics and Bioinformatic Unit- Scientific Direction, IRCCS Regina Elena National

10 Cancer Institute, Via Elio Chianesi 53, 00144, Rome, Italy.

11

12 *Corresponding Author: Marialuisa Appetecchia, marialuisa.appetecchia@ifo.gov.it; Via Elio

13 Chianesi 53, 00144, Rome, Italy 


\section{Abstract}

Background: Gender medicine focuses on how gender differences affect health status and diseases development and how they influence health services access and attitude to screening programmes. Endocrine diseases are influenced by many gender-related issues, some of which have not been sufficiently investigated. The aim of this study was to evaluate gender difference in determinants of health (as lifestyle, level of education, area of origin, distance from hospital) and how these elements could influence diseases prevalence in an endocrine outpatients setting, with a special focus on oncological disease.

Methods: We performed a cross-sectional study enrolling patients referring for the first time to our Oncological Endocrinology Unit, between January 2019 to December 2019.

Results: We enrolled 1107 consecutive patients. Mean age was $56.8 \pm 15.0$ years (77\% females). The main reasons for referral were thyroid and bone diseases. We found a gender difference in some disease prevalences: malignant endocrine diseases and iatrogenic thyroid diseases were more frequent in males, while other thyroid disorders, adrenal and metabolic diseases and cancer treatment induced bone loss were higher in females. The frequency of oncological comorbidities was higher in females. No difference was found in the propensity to travel long distances to reach the hospital. In our population, women had a higher socio-cultural level and followed healthier lifestyle. In fact, alcohol and tobacco consumption was lower in females and women had lower BMI. The percentage of smokers or ex-smokers was higher in patients with any malignancy compared to patients with benign endocrine diseases.

Conclusions: the study showed the importance of considering gender as a determinant of health, able to influence also lifestyle and habits, and as an element to keep in consideration to promote a healthier lifestyle and a targeted endocrine screening especially in oncological setting.

Keywords: gender medicine; lifestyle; endocrine disease; oncological comorbidities; determinants of health. 


\section{Background}

Gender medicine deals with biological, psychological and socio-cultural differences between men and women, which can affect health status and disease development(1). The term "gender" goes beyond the simple biological differences between male and female, classically defined as "sex", taking into account also environmental, social, cultural and relational factors(2).Gender medicine is therefore a complex study of how these gender differences affect the state of health, the onset and progression of diseases, the access to health services, the attitude towards prevention interventions and to therapeutic strategies(3). In fact, men and women, despite being susceptible to almost the same pathologies, could present different symptoms, disease progression and response to treatments. In addition, women have a higher propensity to consult health care providers, generally they take more medication and usually manage family health problems(4). In 2019, Italian Ministry of Health arranged a plan for the application and the diffusion of gender medicine in the country, testifying its importance in the wider field of precision medicine(5).

52 Gender medicine therefore aims to achieve a "health" condition by paying attention not only to the disease itself, but also to the "determinants of health" starting from lifestyles such as alcohol, smoking, physical activity, nutrition and body weight(6). These, in fact, contribute to determining the health of women and men and have an impact on the incidence of many chronic diseases, such as cardiovascular and respiratory diseases, diabetes mellitus, and cancer diseases. Exposure to the aforementioned risk factors depends on individual choices, but it is strongly influenced by the socio-cultural and environmental context and therefore by gender(7). 
59 In modern society, gender medicine appears essential to achieve the best diagnostic and

60 therapeutic work-up for both men and women and to optimize health services planning(8).

61 Endocrinology is one of the medical disciplines most influenced by issues related to gender, as the

62 most widespread endocrine diseases (e.g. thyroid diseases, osteoporosis and diabetes mellitus)

63 have marked gender differences in term of prevalence. This is certainly due to hormonal

64 differences between males and females, while the impact of gender-related determinants of health

65 have not yet been sufficiently investigated(9).

66 The aims of our study were:

67 - to evaluate the gender difference in determinants of health as lifestyle, level of education, area of 68 origin;

69 - to evaluate how and if these determinants could influence the prevalence of endocrine diseases 70 and oncologic disease;

71 - to assess gender difference in availability to travel long distances in order to gain access to 72 health services.

\section{Methods}

This is a cross-sectional study, conducted in our Oncological Endocrinology Unit at Regina Elena National Cancer Institute of Rome, between January 2019 to December 2019. All patients (age > 18 years) referring to our endocrine center, for the first evaluation in the study frame-time, were considered potentially eligible. Patients could gain access to our center through national health system booking service or through selected waiting lists accessible by our hospital's oncologists. 
The following parameters were assessed during first visit: sex, age, residence address to calculate

81 distance from the hospital, ethnicity and nationality, level of education (grade 8 or less: elementary

82 school and middle school; grade 12 or upper education: high school, degree or upper title),body mass index (BMI) calculated as the weight (kilograms) divided by the height (meters squared), smoking status (3 groups: smokers, non-smokers or ex-smokers), alcohol consumption (classified as yes in case of at least $0.5 \mathrm{UI}$ alcohol units per day or no), physical activity (no, yes moderate or yes intense), endocrine and oncological diseases (for example: brain, breast, prostate, lung, gastrointestinal cancers, hemato-lymphoid neoplasms).

Endocrine diseases were grouped as follows: pituitary diseases, andrological diseases, neuroendocrine neoplasms, thyroid cancers, benign thyroid diseases (including thyroid nodules, thyroiditis, primary hyperthyroidism and hypothyroidism, iatrogenic thyroid disorders induced by amiodarone, tyrosine kinase inhibitors-TKI, immune checkpoint inhibitors, neck radiotherapy), bone diseases (including cancer treatment induced bone loss-CTIBL, post-menopausal osteoporosis and osteopenia, hyperparathyroidism and hypercalcemia), adrenal diseases (including adrenal hyperplasia, functioning and non-functioning adrenal adenomas and incidentalomas), diabetes mellitus and metabolic diseases (including diabetes mellitus type 2, obesity, insulin resistance). Each patient's data were collected using a standardized data collection form.

97 The study was done under the approval of the local ethic committee of Regina Elena National

98 Cancer Institute and all patients gave written informed consent to participate. 
100 Variables of interest were expressed as frequencies and percentage values while continuous

101 variables were expressed as mean \pm standard deviation. Patients were divided in subgroups

102 according to gender and age. Associations among variables were tested with non-parametric

103 Chi-square test. Statistically significance was defined at $\mathrm{p}<0.05$. All statistical analyses were

104 performed using Statistical Packages for Social Sciences (SPSS version 21.0).

\section{Results}

\subsection{Patients' characteristics}

111 difference between gender (male: $60 \pm 16.6$ years, female $56 \pm 14.3$ years, $\mathrm{p}<0.001$ ). No gender

112 difference was found in ethnicity of patients. A higher proportion of female patients had a

113 nationality other than Italian ( $\mathrm{p}=0.008,29$ patients from European Union, 5 from South America,

11416 from Asia, 2 from Africa).

\section{Table 1. Patients' characteristics.}

\begin{tabular}{|c|c|c|c|}
\hline & $\begin{array}{c}\text { Male } \\
\mathrm{N}=\mathbf{2 5 3}(\mathbf{2 3 \%})\end{array}$ & $\begin{array}{c}\text { Female } \\
\mathrm{N}=854(77 \%)\end{array}$ & p value \\
\hline Age & $60 \pm 16.6$ & $56 \pm 14.3$ & $<0.001^{*}$ \\
\hline Age $\leq 45$ years & $50(19.9 \%)$ & $202(23.7 \%)$ & $0.209^{\#}$ \\
\hline Age $>45$ years & $201(80.1)$ & $650(73.6 \%)$ & \\
\hline \multicolumn{4}{|l|}{ Ethnicity } \\
\hline Caucasian n (\%) & $250(98.8 \%)$ & $839(98.6 \%)$ & $1.0^{\#}$ \\
\hline Others n (\%) & $3(1.2 \%)$ & $12(1.4 \%)$ & \\
\hline
\end{tabular}




\begin{tabular}{|c|c|c|c|}
\hline \multicolumn{4}{|l|}{ Nationality } \\
\hline Italian & $249(98.4 \%)$ & $806(94.4 \%)$ & $0.008^{* *}$ \\
\hline Others & $4(1.6 \%)$ & $48(5.6 \%)$ & \\
\hline \multicolumn{4}{|l|}{ Level of education } \\
\hline Grade 8 or lower & $86(38.2 \%)$ & $239(30.3 \%)$ & $0.024 * \#$ \\
\hline Grade 12 or upper education & $139(61.8 \%)$ & $551(69.7 \%)$ & \\
\hline \multicolumn{4}{|l|}{ Distance from the hospital } \\
\hline$\leq 10 \mathrm{Km}$ & $51(20.2 \%)$ & $189(22.1 \%)$ & $0.229^{\#}$ \\
\hline $10-20 \mathrm{Km}$ & $79(31.2 \%)$ & $223(26.1 \%)$ & \\
\hline $20-30 \mathrm{Km}$ & $39(15.4 \%)$ & $169(19.8 \%)$ & \\
\hline$>30 \mathrm{Km}$ & $84(33.2 \%)$ & $272(31.9 \%)$ & \\
\hline \multicolumn{4}{|l|}{ Determinants of health } \\
\hline BMI & $27.8 \pm 5.0$ & $26.1 \pm 5.5$ & $<0.001 *$ \\
\hline \multicolumn{4}{|l|}{ Smoking status } \\
\hline Smokers or ex-smokers & $150(59.8 \%)$ & $356(42.0 \%)$ & $<0.001$ *\# \\
\hline No smokers & $101(40.2 \%)$ & $492(58.0 \%)$ & \\
\hline \multicolumn{4}{|l|}{ Alcohol Consumption } \\
\hline No & $125(50.8 \%)$ & $559(67.0 \%)$ & $<0.001$ *\# \\
\hline Yes & $121(49.2 \%)$ & $275(33.0 \%)$ & \\
\hline \multicolumn{4}{|l|}{ Physical activity } \\
\hline No & $139(65.0 \%)$ & $468(63.8 \%)$ & $0.854^{\#}$ \\
\hline Yes, moderate & $69(32.2 \%)$ & $240(32.7 \%)$ & \\
\hline Yes, intense & $6(2.8 \%)$ & $26(3.5 \%)$ & \\
\hline
\end{tabular}

116 Legend and abbreviations: ${ }^{*}=$ statistically significant differences between groups; ${ }^{*}=$ chi-square

117 test. Values are expressed as mean \pm standard deviation if not otherwise statedare cited.

118 3.2. Reason for referral

120 frequent reason was bone disease. Among patients referring for benign endocrine diseases, there

121 was a high prevalence of oncological comorbidity. In fact, at list one malignancy was present in

$12256.1 \%$ of patients with benign endocrine disease compared to only $14.5 \%$ of patients with

123 endocrine malignancies, with a statistically significant difference $(\mathrm{p}<0.001)$. Considering gender, 
124 malignant endocrine diseases were more frequent in males than in females $(11.5 \%$ vs $5.3 \%$,

$125 \mathrm{p}<0.001)$. All reasons for referral are summarized in Table 2.

126 Table 2. Reasons for referral.

\begin{tabular}{|c|c|c|}
\hline & $\begin{array}{c}\text { Male } \\
\mathrm{N}=253(23 \%)\end{array}$ & $\begin{array}{c}\text { Female } \\
\mathrm{N}=\mathbf{8 5 4}(\mathbf{7 7 \%})\end{array}$ \\
\hline Benign thyroid diseases & $147(58.1 \%)$ & $476(55.7 \%)$ \\
\hline Thyroid cancers & $19(7.5 \%)$ & $39(4.6 \%)$ \\
\hline Bone diseases & $22(8.7 \%)$ & $247(28.9 \%)$ \\
\hline Adrenal diseases & $21(8.3 \%)$ & $37(4.3 \%)$ \\
\hline Pituitary diseases & $9(3.6 \%)$ & $14(1.6 \%)$ \\
\hline Neuroendocrine neoplasms & $10(4.0 \%)$ & $6(0.7 \%)$ \\
\hline Diabetes mellitus and metabolic diseases & $9(3.6 \%)$ & $18(2.1 \%)$ \\
\hline Andrological diseases & $9(3.6 \%)$ & NA \\
\hline Others & $7(2.8 \%)$ & $17(2.0 \%)$ \\
\hline
\end{tabular}

127 Values are expressed as number of patients (percentage). NA= not applicable.

\subsection{Disease prevalence according to gender}

Most patients referred to our Unit for just one endocrine disease (corresponding to the reason

130 for referral), while a lower but significant percentage of patients had more than one disease: in

131 particular, 227 patients had two endocrine diseases and 23 patients had 3 endocrine

132 diseases.Global diseases prevalence is summarized in Table 3.The most frequent group of

133 disorders were benign thyroid diseases, without gender difference $(p=0.517)$. In a subgroup

134 analysis, percentage of iatrogenic thyroid disorders (induced by amiodarone, TKI, immune

135 checkpoint inhibitors, neck radiotherapy) was higher in males (10.6\%, vs $1.4 \%$ in females,

$136 \mathrm{p}<0.001$ ), while no statistically significant difference was found in percentage of patients with

137 thyroid nodules according to gender (70\% in males, $69.9 \%$ in females, $\mathrm{p}=0.77)$. Consequently, 
138 females had higher prevalence of remaining thyroid diseases (hypothyroidism and

139 hyperthyroidism, thyroiditis).

140 There was a statistically significant difference in the proportion of females and males affected by

141 bone diseases (respectively, $36.1 \%$ vs10.7\%, $<<0.001$ ); in this group most patients had CTIBL and

142 only a minority of patients had other bone diseases not related to cancer, such as post-menopausal

143 osteoporosis or disorders of calcium metabolism in both gender. Considering CTIBL prevalence,

144 there was a statistically significant difference between males and females $(27.3 \%$ vs $7.1 \%$,

$145 \mathrm{p}<0.001)$.

146 In our population, there was no gender difference in the prevalence of pituitary diseases $(\mathrm{p}=0.288)$,

147 while adrenal and metabolic diseases were higher in females (respectively $\mathrm{p}=0.002$ and $\mathrm{p}=0.024$ ).

148 Considering malignancies, a higher proportion of males referred for neuroendocrine neoplasms

$149(4.0 \%$ vs $0.7 \%, \mathrm{p}<0.001)$ and thyroid cancers $(7.9 \%$ vs $4.7 \%, \mathrm{p}=0.047)$, while females had a higher

150 prevalence of oncological comorbidities than males $(\mathrm{p}=0.005)$. In the subgroup of patients affected

151 by oncological comorbidities, a higher percentage of women had CTIBL than men (45.0\% vs

$15216.1 \%, \mathrm{p}<0.001)$.

153 Table 3. Diseases prevalence

\begin{tabular}{|c|c|c|}
\hline & $\begin{array}{c}\text { Male } \\
\mathrm{N}=253(23 \%)\end{array}$ & $\begin{array}{c}\text { Female } \\
\mathrm{N}=854(77 \%)\end{array}$ \\
\hline Benign thyroid diseases & $160(63.2 \%)$ & $559(65.5 \%)$ \\
\hline Thyroid cancers & $20(7.9 \%)$ & $40(4.7 \%)$ \\
\hline Bone diseases & $27(10.7 \%)$ & $308(36.1 \%)$ \\
\hline Adrenal diseases & $23(9.1 \%)$ & $35(4.1 \%)$ \\
\hline Pituitary diseases & $9(3.6 \%)$ & $20(2.3 \%)$ \\
\hline Neuroendocrine neoplasms & $10(4.0 \%)$ & $6(0.7 \%)$ \\
\hline Diabetes mellitus and metabolic diseases & $27(10.7 \%)$ & $55(6.4 \%)$ \\
\hline Andrological diseases & $9(3.6 \%)$ & NA \\
\hline
\end{tabular}




\begin{tabular}{|l|c|c|}
\hline Others & $7(2.8 \%)$ & $19(2.2 \%)$ \\
\hline Oncological comorbidities & $115(45.5 \%)$ & $474(55.5 \%)$ \\
\hline
\end{tabular}

154 This Table takes in consideration all patients diseases. Values are expressed as number of patients

155 (percentage). NA= not applicable.

\subsection{Gender-related determinants of health}

No difference was found in physical activities between males and females $(\mathrm{p}=0.854)$, and this

158

159

160

161 ( $<<0.001)$; accordingly, 58\% of women had never smoked in their life compared to only $42 \%$ of

162 males. Dividing the study population in two groups according to age, in younger people there was

163 no statistically significant difference in smoking habits regarding to sex, while in people older than

16445 years there was a statistically significant gender difference in the proportion of smokers

165 ( $\mathrm{p}<0.001)$. Also alcohol consumption was higher in males than in females (49.2 vs $33.0 \%$,

$166 \mathrm{p}$ <0.001). As smoking habits, there was a difference in alcohol habits only in older

167 patients $(\mathrm{p}<0.001)$.

168 No difference was found in smoking status, physical activity and alcohol consumption regarding

169 nationality (comparing Italian and foreign patients), neither between patients referring for benign

170 and malignant endocrine diseases. However, gathering together people with any malignancies,

171 both endocrine or non-endocrine, the percentage of smokers or ex-smokers was higher compared

172 to the group without malignancies $(\mathrm{p}=0.041)$, while no difference in proportion was found

173 regarding physical activity and alcohol consumption. 
174 In our study population, women had a higher level of education compared to men ( $\mathrm{p}=0.024)$, as

175 well as not Italian people had more frequently a degree or upper title compared to Italian patients

$176 \quad(39.1 \%$ vs $23 \%, \mathrm{p}=0.02)$.

177 Only $22 \%$ of patients lived nearby the hospital $(<10 \mathrm{~km})$. The prevalence of malignant endocrine

178 disease was $7.7 \%$ in the group of patients who lived far from the hospital $(>10 \mathrm{~km})$ versus $3.8 \%$ in

179 patients who lived closer $(\mathrm{p}=0.031)$. No significant difference was found in the distance from the

180 hospital according to gender, nationality or non-endocrine oncological comorbidities.

181 Gender-related determinants of health are summarized in Table 1.

\section{Discussion}

183 Gender medicine has recently received increasing attention(10). In this perspective, we

184 decided to design this cross-sectional study in order to provide an overview of our patients,

185 focusing on the role of gender on endocrine diseases, risks factors and other important aspects

186 related to health care, always in a gender perspective. Many studies in the literature have focused

187 on the impact of gender on lifestyle(11). Men seem more prone to consume alcohol and to develop

188 alcohol-related diseases compared to women(12). Conversely, women who physiologically

189 tolerate lower amount of alcohol (due to the sex differences in gastric absorption and metabolism),

190 usually drink less alcohol also for cultural reason, as society's disapproval of drinking or increased

191 risk of physical and sexual assault $(12,13)$.

192 In our population, we confirmed a gender difference in alcohol consumption, which was lower in

193 females. Interestingly, this difference was not statistically significant in younger people, testifying

194 as younger women have a more similar lifestyle to males, perhaps due to female emancipation, 

proportion of men was smokers than women. In Italy, data from two recent tobacco use surveys

197 show a smoking prevalence of $26 \%$ in men compared to $17.2 \%$ in women. This gender difference is

198 reduced in young adults: in Italian adolescents (15-24 years), 21.9\% of boys are smokers against

$19918.2 \%$ of girls(14). Therefore, these studies underline that the use of tobacco in young women is a 200 behavior to be monitored carefully. The relevant gender difference in tobacco use has been 201 confirmed by Italian Ministry of Health, in a Tobacco Prevention and Control Report based on 202 ISTAT data: the estimated smoking prevalence is $19.8 \%$ (24.8\% males and $15.1 \%$ females)(15). 203 These data are consistent with our results: in the whole study population, men were more 204 frequently smokers or ex-smokers compared to women but, considering only patients aged 45 or 205 less (born after Italian women emancipation) this difference has not been confirmed, testifying to a 206 change in lifestyle in new generations. Several factors should be considered in the relationship 207 between smoking and female gender. Low sociocultural and educational levels and living in 208 developing countries are known to be risk factors for the onset of tobacco consumption(16, 17). In 209 the last years the proportion of women who become smoker has increased, mainly due to women's 210 earning power and targeted marketing by tobacco companies(18).

211 Scientific research has also shown differences in food intake and the practice of physical activity in 212 both sexes(19, 20). For example, in modern Western societies, the male gender seems to prefer red 213 meat, high protein foods and sugar-sweetened beverages, while healthier foods such as vegetables, 214 fruit, fish and dairy products are mostly eaten by women(20-22). These differences may depend on 215 a different awareness of the relationship between food behavior and health and on a different 
216 attention to weight control or good physical shape, in line with modern society stereotypes(23).

217 This attitude is reflected in the nutritional pattern and body mass index. Unfortunately, we did not

218 collect information on dietary habits of our patients, but males had a higher BMI compared to

219 women, testifying to probably less healthy dietary habits. In our population, there were no

220 differences in physical activity level between males and females, unlike other studies published in

221 literature which have shown, especially in younger people, a greater propensity to physical

222 activities in males than in females $(24,25)$.

223 Taking all these aspects together, in our study population, women seem to follow a healthier

224 lifestyle. This data could dependon the influence of multiple factors. First, women pay greater

225 attention to their health condition compared to men(4); secondary, it is the conditioning of society

226 that leads women to maintain a good body shape in order to achieve beauty stereotypes; finally, the

227 level of education of patients. Indeed, in our population, women had a higher level of education

228 compared to men and this could have affected the lifestyle of our patients. It is demonstrated that

229 better educated people follow a healthier lifestyle, probably due to the increased awareness of the

230 correlation between lifestyle and health(26).

231 National habits also seem to influence the state ofhealth; in fact, in our study, foreign people

232 (mainly women from European Union States) did not follow a healthier lifestyle than Italian

233 patients, despite their higher education level.

234 During a year, the percentage of women referring for a first endocrine visit was significantly higher

235 than men. This fact could be explained by the more common prevalence of endocrine diseases in 
236 females(27), but also by the higher attention paid to personal health status typical of women and

237 their higher propensity to refer to health care centers(1).

238 One of the aims of our study was to ascertain if women were more willing than men to travel long

239 distances for obtaining medical care. The willingness to move from own home area to reach

240 tertiary center health care was the same in both sexes and did not change according to nationality;

241 in fact, most patients decided to go to a qualified cancer endocrinology center, even if it was not

242 close to their residences. This was particularly true for patients with endocrine tumors who showed

243 a higher propensity to travel long distances. It is important to consider that a relevant part of the

244 first access to our center was allowed regardless of the reported disease, considering that the

245 booking service of the Italian public health system does not indicate to the patients the most

246 suitable hospital. According to this, the reason for referral to our center was only partially biased

247 by the type of center (Oncological Endocrine Unit).

248 The most common reason for referral to our center was an endocrine malignancy or a benign

249 endocrine disease associated to other oncological comorbidities, probably because our center is a

250 national reference hospital for neoplastic diseases. In our population, women had a higher

251 prevalence of oncological comorbidities: a high percentage of these patients referred to the

252 Endocrine Unit for prevention and treatment of cancer induced bone loss, mainly due to the intake

253 of aromatase inhibitors or gonadotropin-releasing hormone analogs for breast cancers.

254 Benign thyroid diseases were the most common endocrine disorders in both sexes; this finding

255 could be explained by the high prevalence of thyroid nodules. The increased prevalence in our

256 population could be caused by the fact that oncological patients undergo multiple and deepened 
257 whole body radiological examinations (such as computed tomography and magnetic resonance

258 imaging) and functional procedures (such as positron emission tomography), therefore thyroid

259 nodules can be an accidental finding(28), and patients have been referred to our Unit for

260 subsequent tests, such as the fine needle aspiration diagnostic biopsy of the thyroid nodule.

261 Furthermore, there was no gender difference in the prevalence of benign thyroid disease, although

262 some disorders, such as Hashimoto's thyroiditis or thyroid nodules, are known to be more frequent

263 in females(27). However, this finding could be explained considering that in our population this

264 group of diseases also contains drug related thyroid dysfunction (e.g. due to TKI or amiodarone

265 intake) or subclinical thyroid disease due to age or chronic diseases, which usually do not showed

266 significant gender differences(29, 30).

267 Probably, the high incidence of adrenal incidentalomas could be explained by the high number of

268 radiological and functional tests performed in our Hospital for the cancer diagnosis and for

269 patients' follow-up.

270 In our study, thyroid malignancies were more frequent in males than females.The risk of

271 malignancy of the thyroid nodules is known to be greater in males $(31,32)$, and that male gender is

272 an independent prognostic factors in papillary thyroid carcinoma, which influences staging and

273 risk of recurrence $(33,34)$. These factors together could explain the propensity to refer to a national

274 cancer center in male patients.

275 We therefore sought to evaluate the impact of common gender-influenced risk factors in the higher

276 prevalence of endocrine malignancies found in males. 
277 In patients with at least one tumor (endocrine or non-endocrine), the percentage of smokers and

278 ex-smokers was higher compared to patients without neoplasms.

279 This finding has not been confirmed considering only patients with endocrine malignancies, 280 probably due to the low number of patients affected and also considering that for some of these 281 malignancies (for example thyroid cancer) other risk factors are of considerable importance, such 282 as family history or previous radiation exposure(32, 35).

283 In a gender medicine perspective, it should be also interesting to evaluate possible differences 284 between men and women in terms of inclination in carrying out periodic follow-up visits and 285 adherence to the treatment proposed by physician, with possible impact on progression and 286 outcomes of the endocrine disorders. Unfortunately, we do not have data on these aspects in our 287 population, as only one visit per patient has been analysed, but this topic is of a great interest for 288 further studies.

\section{Conclusions}

Biological and sociocultural aspects are known to influence lifestyle, patient health, disease

291 development and treatment adherence. The study highlights and confirms the importance of 292 considering gender and gender-related health determinants as key factors for health, even in 293 patients affected by endocrine diseases, in which this approach has not been widely used.

294 Our study demonstrated that smoking and alcohol consumption are more common in males and 295 younger women. Therefore, in the approach to the patient, doctors should pay special attention to 296 female and male lifestyle, in order to discourage voluptuous habits and to encourage physical 297 activity and healthy eating habits. This is particularly important mainly in subjects who, nowadays, 
298 seem to be less careful to these aspects, as men, younger women or patients with lower

299 socio-cultural level.

300 Our study confirmed, as already known in the literature, that women were more affected by

301 endocrine disorders but, in our cohort, the proportion of endocrine malignancies was higher in

302 men. Therefore, from a precision medicine perspective, all efforts must be made to raise

303 awareness, in oncological patients and health care providers, on the risk of endocrine diseases

304 development in order to promote targeted screening in both genders.

305 List of abbreviations:

306 TKI: tyrosine kinase inhibitors; CTIBL: cancer treatment induced bone loss; BMI: body mass 307 index.

\section{Declarations}

309 Ethics approval and consent to participate

310 The study has been approved by the local ethic committee of Regina Elena National Cancer

311 Institute (reference number: 1370/20). All patients give written informed consent to study

312 participation.

\section{Availability of data and materials}

314 The datasets generated during the current study are available in the GARR repository,

315 [https://www.garr.it/it/]

\section{Competing interests}

317 The authors declare they have no competing interests. 


\section{Funding}

Not applicable

\section{Author Contributions}

MB: Study conduction, data collecting, initial data analysis, writing - original draft. GP: Data control, initial data analysis, writing - original draft. AC, RL, MM, AB: Data collecting. IT: Statistical analysis. M.A.: study conception and design, supervision, writing - review \& editing. All authors have read and agreed to the published version of the manuscript.

\section{References}

1. McGregor AJ, Templeton K, Kleinman MR, Jenkins MR. Advancing sex and gender competency in medicine: sex \& gender women's health collaborative. Biol Sex Differ. 328 2013;4(1):11.

329 2. Ristvedt SL. The evolution of gender. JAMA Psychiatry. 2014;71(1):13-4.

330 3. Legato M. Principles of Gender-Specific Medicine. 2nd Edition. Elsevier. 2011.

331 4. Cortese DA. A vision of individualized medicine in the context of global health. Clin 332 Pharmacol Ther. 2007;82(5):491-3.

333 5. http://www.salute.gov.it/imgs/C_17_pubblicazioni_2860_allegato.pdf.

334 6. Vlassoff C. Gender differences in determinants and consequences of health and illness. J 335 Health Popul Nutr. 2007;25(1):47-61.

336 7. Legato MJ, Johnson PA, Manson JE. Consideration of Sex Differences in Medicine to 337 Improve Health Care and Patient Outcomes. JAMA. 2016;316(18):1865-6.

338 8. Peres A. Gender-specific medicine in clinical research and health policy implications. Italian 339 Journal of Gender-Specific Medicine. 2018;4(2):45-6.

340 9. Lauretta R, Sansone M, Sansone A, Romanelli F, Appetecchia M. Gender in Endocrine 341 Diseases: Role of Sex Gonadal Hormones. Int J Endocrinol. 2018;2018:4847376.

342 10. Baggio G. [Gender medicine: a interdisciplinary approach to medicine.]. G Ital Med Lav 343 Ergon. 2017;39(3):196-8.

344 11. Vari R, Scazzocchio B, D'Amore A, Giovannini C, Gessani S, Masella R. Gender-related 345 differences in lifestyle may affect health status. Ann Ist Super Sanita. 2016;52(2):158-66.

346 12. Erol A, Karpyak VM. Sex and gender-related differences in alcohol use and its consequences: 347 Contemporary knowledge and future research considerations. Drug Alcohol Depend. $348 \quad 2015 ; 156: 1-13$.

349 13. Agabio R, Pisanu C, Gessa GL, Franconi F. Sex Differences in Alcohol Use Disorder. Curr 350 Med Chem. 2017;24(24):2661-70. 

2015-2016: prevalence, trends, roll-your-own cigarettes, and attitudes towards incoming regulations. Tumori. 2017;103(4):353-9. 15. http://www.istat.it/it/files/2016/12/Asi-2016.pdf.

16. Giskes K, Kunst AE, Benach J, Borrell C, Costa G, Dahl E, et al. Trends in smoking behaviour between 1985 and 2000 in nine European countries by education. J Epidemiol Community Health. 2005;59(5):395-401.

17. Harrell JS, Bangdiwala SI, Deng S, Webb JP, Bradley C. Smoking initiation in youth: the roles of gender, race, socioeconomics, and developmental status. J Adolesc Health. 1998;23(5):271-9. 18. Shafey O, Fernandez E, Thun M, Schiaffino A, Dolwick S, Cokkinides V. Cigarette advertising and female smoking prevalence in Spain, 1982-1997: case studies in International Tobacco Surveillance. Cancer. 2004;100(8):1744-9. 19. Li KK, Concepcion RY, Lee H, Cardinal BJ, Ebbeck V, Woekel E, et al. An examination of sex differences in relation to the eating habits and nutrient intakes of university students. J Nutr Educ Behav. 2012;44(3):246-50.

20. Rolls BJ, Fedoroff IC, Guthrie JF. Gender differences in eating behavior and body weight regulation. Health Psychol. 1991;10(2):133-42.

21. Fagerli RA, Wandel M. Gender differences in opinions and practices with regard to a "healthy diet". Appetite. 1999;32(2):171-90.

22. Yahia N, Wang D, Rapley M, Dey R. Assessment of weight status, dietary habits and beliefs, physical activity, and nutritional knowledge among university students. Perspect Public Health. 2016;136(4):231-44.

23. Ferguson C, Winegard B, Winegard B. Who Is the Fairest One of All? How Evolution Guides Peer and Media Influences on Female Body Dissatisfaction. Rev Gen Psychol 2011;15(1):11-28. 24. Salles-Costa R, Heilborn ML, Werneck GL, Faerstein E, Lopes CS. [Gender and leisure-time physical activity]. Cad Saude Publica. 2003;19 Suppl 2:S325-33. 25. Solmon MA. Physical education, sports, and gender in schools. Adv Child Dev Behav. 2014;47:117-50.

26. Brobeck E, Bergh H, Odencrants S, Hildingh C. Lifestyle advice and lifestyle change: to what degree does lifestyle advice of healthcare professionals reach the population, focusing on gender, age and education? Scand J Caring Sci. 2015;29(1):118-25. 28. Russ G, Leboulleux S, Leenhardt L, Hegedus L. Thyroid incidentalomas: epidemiology, risk stratification with ultrasound and workup. Eur Thyroid J. 2014;3(3):154-63. amiodarone-induced thyrotoxicosis: Update and review of the literature. Ann Endocrinol (Paris). 2019;80(1):54-60. changes in thyroid function in the oldest old and survival: the cardiovascular health study all-stars study. J Clin Endocrinol Metab. 2012;97(11):3944-50.

391 31. Mettler J, Armefti S, Schmidt M, Faust M, Engels M, Chiapponi C. Benign Thyroid Diseases: 
393 Nodules? Results from a 4-Year Retrospective Analysis of an Endocrine Tumor Board. Visc Med. 394 2020;36(1):28-33.

395 32. Gharib H, Papini E, Garber JR, Duick DS, Harrell RM, Hegedus L, et al. American 396 Association of Clinical Endocrinologists, American College of Endocrinology, and Associazione 397 Medici Endocrinologi Medical Guidelines for Clinical Practice for the Diagnosis and Management 398 of Thyroid Nodules--2016 Update. Endocr Pract. 2016;22(5):622-39.

399 33. Kao YH, Gan HK, Zaheer S, Lam WW, Loke KS, Wong WY, et al. Gender, Race, and Age at 400 Diagnosis as Risk Factors for Metastasis or Recurrence among 1,657 Thyroid Cancer Patients 401 Treated with Radioiodine across 40 Years in Singapore. Oncol Res Treat. 2015;38(12):679-82.

402 34. Ding J, Wu W, Fang J, Zhao J, Jiang L. Male sex is associated with aggressive behaviour and 403 poor prognosis in Chinese papillary thyroid carcinoma. Sci Rep. 2020;10(1):4141.

404 35. Su X, Li Z, He C, Chen W, Fu X, Yang A. Radiation exposure, young age, and female gender 405 are associated with high prevalence of RET/PTC1 and RET/PTC3 in papillary thyroid cancer: a 406 meta-analysis. Oncotarget. 2016;7(13):16716-30.

407 